\title{
Simulation and Sensitivity Analysis of Molybdenum Disulfide Nanoparticle Production Using Aspen Plus
}

\author{
Mouad Hachhach (D), Hanane Akram (D, Mounir Hanafi, Ouafae Achak, and Tarik Chafik \\ Laboratory of Chemical Engineering and Valorization of Resources, LGCVR-UAE/U14FST, \\ Faculty of Sciences and Techniques of Tangier, Abdelmalek Essaadi University, P.O. Box 416, Tangier, Morocco
}

Correspondence should be addressed to Mouad Hachhach; mhachhach@uae.ac.ma and Hanane Akram; akramhanane@yahoo.fr Received 17 May 2019; Revised 24 August 2019; Accepted 31 August 2019; Published 28 October 2019

Academic Editor: Prem Kumar Seelam

Copyright ( $) 2019$ Mouad Hachhach et al. This is an open access article distributed under the Creative Commons Attribution License, which permits unrestricted use, distribution, and reproduction in any medium, provided the original work is properly cited.

\begin{abstract}
The sensitivity analysis of molybdenum disulfide nanoparticles synthesis process is studied using Aspen Plus with the aim of investigating the effect of reactants' amounts on the production of molybdenum disulfide nanoparticles. The adopted approach consists in simulating the synthesis process based on experimental data, obtained at laboratory scale followed by sensitivity analysis with respect to the following precursors: ammonium heptamolybdate, elemental sulfur, and hydrazine used as a reducing agent. The sensitivity analysis revealed that the precursors have more significant impact on the obtained amount of molybdenum disulfide compared to hydrazine. The obtained result showed that the approach adopted in the study might be of interest for further investigation of the process design and scaling-up.
\end{abstract}

\section{Introduction}

The solvothermal synthesis is among the widely used methods for nanomaterials production through chemical reactions in a closed system involving appropriate solvent at temperatures higher than its boiling point. The technique was inspired from a natural production of many minerals in the earth's crust under the conditions of high temperature and pressure permitting the formation of many minerals [1]. This technique was used afterward for the production of innovative nanomaterials of interest for different fields, such as catalysis [2-8] and environment protection and energy [9-11], as well as medicine [12] and mechanics [13]. As known, nanomaterials are obtained using various methods following bottom-up and top-down approaches [14]. The top-down method consists in breaking down large pieces of material to generate the required nanostructures, whereas bottom-up approach implies assembling single atoms and molecules into larger nanostructures. The bottom-up approach is more advantageous than the top-down approach with respect to nanomaterial production, particularly, the solvothermal method. Its application was found to be efficient for obtaining a variety of structures, sizes, and morphologies that drew attention for applications in heterogeneous catalysis, electrochemistry, and tribology [1517]. On the other hand and as reported in our previous work, the solvothermal approach proves to be more eco-friendly in comparison with other technics [18].

Among the most studied nanomaterials, molybdenum disulfide has shown interesting properties regarding various applications, among which it is used as wind turbines lubricants additive [19]. As reported in our previous work, the preparation of molybdenum disulfide via the solvothermal method is quite easy and consists in mixing reactants in a Teflon-lined stainless steel autoclave filled with a solvent up to $40 \%-80 \%$ of its total volume [8]. The autoclave is maintained at an appropriate temperature and time needed to allow reaction process to occur following either one or two steps. The use of surfactant, phase transferring agent, or templates such as silicon dioxide was found to help the control of molybdenum disulfide nanomaterials size and shape [20-22]. Indeed, the micellar structures are formed from self-assembling of surfactant molecules and yield to nanometre size droplets with insoluble cores and soluble 
shells. The process provides an interesting template contribution as nanoreactors for the synthesis of nanoparticles with controllable diameter. Thus, selection of appropriate surfactant type and amount is required, considering their determining role with respect to the chemical composition of the final product $[23,24]$. On the other hand, even though the surfactants have the ability to help in the growth of nanoparticles as well as their size and shape, the obtained materials might exhibit nonhomogeneity in their chemical composition and crystallinity [13]. Hence, solvothermal synthesis proceeds generally, via three steps: first, the formation of a supersaturated solution, then nucleation, and finally nuclei growth. Note that the powder formation is controlled also by fluctuations phenomena in the supersaturated solution occurring during synthesis process [11]. Thus, it is important to study the correlation of pressure and temperature as well as the nature of solvent that affects the dielectric constants permitting higher supersaturation degree, in addition to the role of enhanced nucleation sites and rate, occurring under supercritical conditions. So far less data are available to provide comprehensive understanding of molybdenum disulfide solvothermal synthesis mechanism. Also, further kinetic and thermodynamic data are still lacking so as to help the scaling-up study. Nevertheless, this issue can be addressed through process simulation making use of available data obtained at laboratory scale. The approach can be of interest with respect to cost estimation and evaluation of the potential of process transfer at industrial scale, as reported in several studies $[25,26]$.

In this regard, the use of Aspen Plus V9 can be of interest considering its ability to model complex processes using simple graphical interface [27]. The software allows, also, the use of available broad database of chemicals and physical parameters that permit built-in operation units suitable for sensitivity analysis and modelling of various processes related to petrochemicals [28], catalysts [29], hydrogen production [30], and carbon dioxide capture [31]. The application of Aspen Plus was reported in the field of nanotechnology [32, 33]; however, it should be pointed out that, to the best of our knowledge, this approach has never been used for investigating such nanomaterials synthesis. Hence, the present work is devoted to sensitivity analysis and simulation of large-scale production of molybdenum disulfide nanoparticles, using Aspen Plus. The study is carried out based on our data concerning the solvothermal preparation of molybdenum disulfide nanoparticles under mild conditions at laboratory scale [17].

\section{Materials and Methods}

2.1. Experimental. For the synthesis technique adopted in this work, we have used the one-pot solvothermal process developed by Akram et al. allowing the preparation of welldispersed molybdenum disulfide nanospheres [17]. The used reagents were purchased from Sigma-Aldrich and used without further purification (Table 1). The synthesis process is as follows: putting $0.14 \mathrm{~g}$ of ammonium heptamolybdate $\left(\left(\mathrm{NH}_{4}\right)_{6} \mathrm{Mo}_{7} \mathrm{O}_{24}, \mathrm{H}_{2} \mathrm{O}\right), 0.05 \mathrm{~g}$ of elemental sulfur $(\mathrm{S}), 1.15 \mathrm{~g}$ of lithium hydroxide $\left(\mathrm{LiOH} \cdot \mathrm{H}_{2} \mathrm{O}\right), 0.06 \mathrm{~g}$ of ammonium carbonate $\left(\left(\mathrm{NH}_{4}\right)_{2} \mathrm{CO}_{3}\right)$, and $8 \mathrm{ml}$ of hydrazine $\left(\mathrm{N}_{2} \mathrm{H}_{4} \cdot \mathrm{H}_{2} \mathrm{O}\right)$ in a Teflon-lined stainless steel autoclave. Ethylenediamine was used (as solvent) to fill the autoclave up to $80 \%$ of its total volume $(200 \mathrm{ml})$. The autoclave was kept at $180^{\circ} \mathrm{C}$ during $24 \mathrm{~h}$. Finally, the solid phase (nanoparticles) is recovered by centrifugation, washed with water and acetone, and then dried under vacuum at $60^{\circ} \mathrm{C}$ for $3 \mathrm{~h}$. The choice of Ethylenediamine as solvent is justified by its ability to remain liquid, under the solvothermal conditions allowing reactants solubilisation. This is due to its appropriate polarity enabling better nucleation and growth of molybdenum disulfide nanoparticles, helping better control of their morphology and their sizes $[15,34]$.

Further details of the used equipment for the synthesis, drying, and recovery of molybdenum disulfide nanoparticles are presented in Table 2.

As reported, the synthesis of molybdenum disulfide nanoparticles is assumed to occur following three steps [15]:

(i) Formation of a supersaturated solution as a result of reactants decomposition in the solvent under the aforementioned operating conditions

(ii) Nucleation and production of stables nuclei

(iii) Growth of the nuclei

2.2. Process Simulation. The adopted approach for the process simulation consists in using purpose-built software to define a system with interconnected components that are solved in either steady state or dynamic mode. Note that the software uses thermodynamic models available in the database and processes flow sheet diagrams so that the various involved unit operations are appropriately positioned and connected. This allows obtaining stable operating point of system behaviour with respect to balance calculation.

Generally, for process modelling, the adopted approach consists in choosing the property method before the creation of the process flowsheet and, then, solving it using Aspen Plus. Note that the choice of property method is crucial because of its influence on the accuracy of material and energy balances [35]. This choice depends on process parameters, such as reactants' amounts, temperature, and pressure in addition to other relevant parameters available in the software database.

In the present study, the ELECNRTL model and the Redlich-Kwong state equations were used together to calculate the involved mixture properties. The ELECNRTL model has been applied mainly in electrolyte systems to represent the solid-liquid equilibrium (SLE) of aqueous multicomponent [36] and phase equilibrium of the mixture of solvents in a wide temperature range [37], while Redlich-Kwong equation is used for vapour phase properties [38].

Flow sheeting is a complete description of material and energy streams needed for process simulation of interest for sizing calculation and cost estimation of chemical process $[39,40]$. The flowsheet creation starts by selecting involved operation units and related equipment interconnects integration within a working system. Accordingly, the flow 
TABLE 1: Summary of the amount and the role of all used reagents.

\begin{tabular}{lccc}
\hline Reagent & Amount & Role & CAS number \\
\hline Ammonium heptamolybdate $\left(\left(\mathrm{NH}_{4}\right)_{6} \mathrm{Mo}_{7} \mathrm{O}_{24}, \mathrm{H}_{2} \mathrm{O}\right)$ & $0.14 \mathrm{~g}$ & Precursor & $12054-85-2$ \\
Sulfur $(\mathrm{S})$ & $0.05 \mathrm{~g}$ & Precursor & $7704-34-9$ \\
Lithium hydroxide $\left(\mathrm{LiOH} \cdot \mathrm{H}_{2} \mathrm{O}\right)$ & $1.15 \mathrm{~g}$ & Strong base & $1310-65-2$ \\
Ammonium carbonate $\left(\left(\mathrm{NH}_{4}\right)_{2} \mathrm{CO}_{3}\right)$ & $0.06 \mathrm{~g}$ & Electrolyte & $506-87-6$ \\
Hydrazine $\left(\mathrm{N}_{2} \mathrm{H}_{4} \cdot \mathrm{H}_{2} \mathrm{O}\right)$ & $8 \mathrm{ml}$ & Reducing agent & $7803-57-8$ \\
Ethylenediamine & $144 \mathrm{ml}$ & Solvent & $107-15-3$ \\
\hline
\end{tabular}

TABLE 2: Summary of brand and models of instruments used in the synthesis of molybdenum disulfide.

\begin{tabular}{|c|c|c|}
\hline Instrument & Brand & Model \\
\hline $\begin{array}{l}\text { Teflon lined } \\
\text { stainless steel autoclave }\end{array}$ & Parr instrument & $4748 \mathrm{~A}$ \\
\hline Centrifuge & Hermal & Z356k \\
\hline $\begin{array}{l}\text { Synthesis } \\
\text { oven }\end{array}$ & Binder & ED35 \\
\hline $\begin{array}{l}\text { Vaccum dryer } \\
\text { oven }\end{array}$ & Thermo scientific & Heraeus VT6025 \\
\hline
\end{tabular}

diagram of the overall process describing the studied molybdenum disulfide nanoparticles synthesis is shown in Figure 1 while the corresponding flowsheet obtained using Aspen Plus is given in Figure 2.

As shown, the process involves two main operation units corresponding to the reactor and the separator. Further descriptions of the major blocks of process flowsheet and the involved streams are given in Tables 3 and 4.

So far, it is assumed for the studied process that the reaction occurs in a stoichiometric reactor "RStoich," chosen among the seven built-in models provided by Aspen Plus database. This reactor seems to fit better the used autoclave device and usually selected when relevant kinetic data are lacking [41]. The input flows correspond to reagents and solvent, whereas the output flows are associated with products and by-products, which are obtained following the separation steps that involve centrifugation, washing, and drying in vacuum in order to separate the solid phase (molybdenum disulfide nanoparticles) from liquid phase (Waste).

In Aspen Plus, the centrifuge is modelled as CFuge block located at the reactor outlet. The output flow contains two flows corresponding to liquid phase (C-waste) and the output stream (C-output) of centrifugation and input of separator block SEP, simulating the washing and drying units. The pure molybdenum disulfide is contained in the Product stream, while all the other impurities are in the Waste stream.

The sensitivity analysis was carried out to study the effect of the variation of the amount of some reactants (precursors and hydrazine) on the final molybdenum disulfide nanoparticle production. Thus, according to base-case requirement, the adopted approach consists in changing the amounts of each reactant (starting with ammonium heptamolybdate, sulfur, and then hydrazine) while keeping the amount of the other reactants and the experimental conditions unchanged, and, afterwards, the amounts of two reactants were changed simultaneously.

\section{Results and Discussion}

The composition of the involved different streams, as simulated by Aspen Plus, is summarized in Table 5. The results show that the synthesized molybdenum disulfide with by-products is present in the reactor output. The centrifugation allows separation of almost all of the liquid phase containing ethylenediamine from the solid phase containing the synthesized nanoparticles, which are subsequently purified after multiple washing and drying steps.

The amount of molybdenum disulfide estimated by simulated process was about $100 \mathrm{mg}$, which is in agreement with the experimentally obtained data at laboratory scale using similar reagents amount and operating conditions [17]. Moreover, the simulation revealed that about $78 \%$ of the initial amounts of both precursors (sulfur and ammonium heptamolybdate) reacted during the synthesis process. Also, it is shown that very small amount of hydrazine $(0.5 \%)$ was involved in the simulated process, which is in agreement with its role as reducing agent. Indeed, under solvothermal conditions, the use of hydrazine generates gaseous species (ammonia, nitrogen) [42], which may lead to an autogenously pressure inside the reactor as a result of supersaturation phenomena. In this regard, it should be pointed out that specific attention must be given to the use of hydrazine because of its instability due to its low autoignition temperature and low explosive limits, in addition to its severe toxicity. On the other hand, the solvent ethylenediamine is almost completely separated by centrifugation process with recovery value about $98 \%$ (Table 5), which agrees with the experimental result. This is due to the fact that the used solvent remains liquid under the operating conditions making its recuperation easier for further recycling.

The process sensitivity analysis, investigated using Aspen Plus, is considered as an important task usually carried out prior to the scaling-up study, so as to assess the effect of the amounts of precursors and reducing agent on the final product yield [43]. As mentioned earlier, the amount of each precursor was changed in the input stream, starting first with ammonium heptamolybdate, then sulfur, and finally hydrazine. As shown in Figures 3 and 4, the variation of the precursor and hydrazine amount yields to a linear increase of the molybdenum disulfide production until reaching a stable maximal value. This maximum amount of molybdenum disulfide obtained is equal to $0.13 \mathrm{~g}$ for $0.18 \mathrm{~g}$ ammonium heptamolybdate, $0.10 \mathrm{~g}$ for $0.04 \mathrm{~g}$ of sulfur, and $0.10 \mathrm{~g}$ for $0.03 \mathrm{~g}$ of hydrazine while maintaining the same amount of the other reagents. 


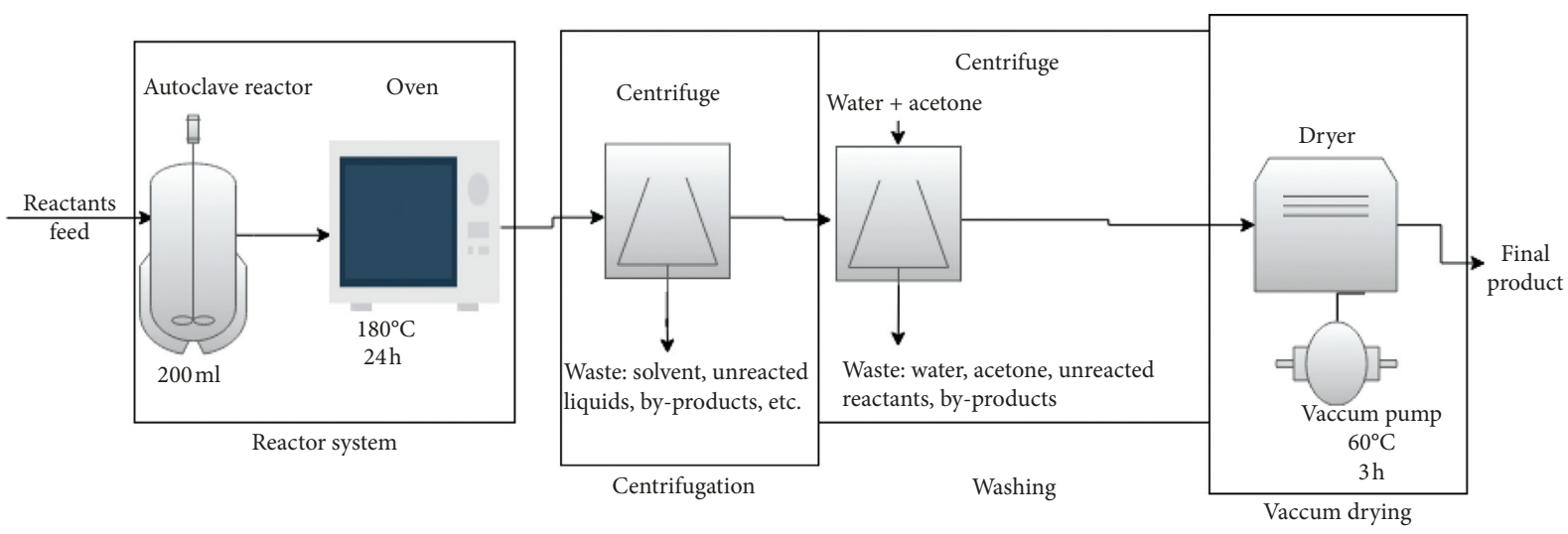

FIGURE 1: Flow diagram of the molybdenum disulfide nanoparticles solvothermal synthesis process at laboratory scale.

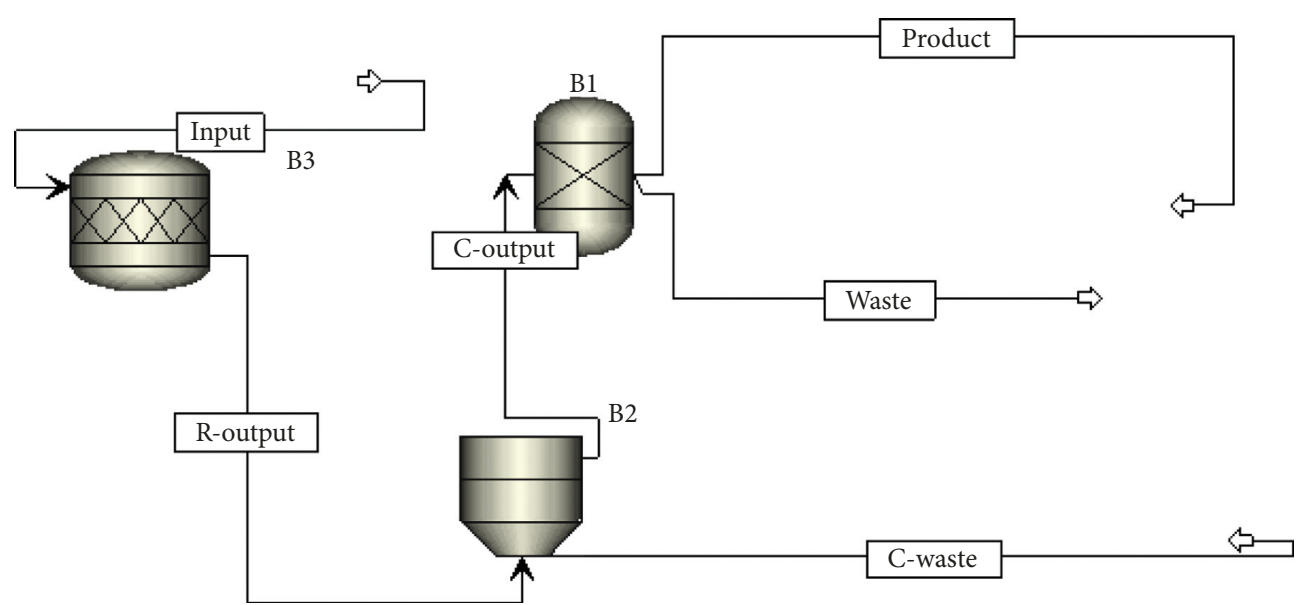

Figure 2: Flowsheet created for molybdenum disulfide synthesis process using Aspen Plus (Snapshot).

TABle 3: Description of the blocks of the created flowsheet.

\begin{tabular}{lc}
\hline Block & Description \\
\hline B1: separator & Separator of components into two streams: Product \\
and Waste & $\begin{array}{c}\text { and phase of r-output stream from } \\
\text { B2: centrifuge }\end{array}$ \\
B3: stoichiometric reactor & Separation of liquid phase \\
\hline
\end{tabular}

TABle 4: Description of the flow sheet streams.

\begin{tabular}{|c|c|}
\hline Stream & Description \\
\hline Input & $\begin{array}{l}\text { Input stream containing } \\
\text { the reagents }\end{array}$ \\
\hline r-output & $\begin{array}{l}\text { Output of the reactor, composed of solution } \\
\text { containing the final product feeding the } \\
\text { centrifugation }\end{array}$ \\
\hline C-output & $\begin{array}{l}\text { Output stream of centrifugation and input of } \\
\text { separator. It contains the solid phase with small } \\
\text { amount of liquid phase }\end{array}$ \\
\hline C-waste & $\begin{array}{c}\text { Stream of the liquid phase containing mainly the } \\
\text { solvents }\end{array}$ \\
\hline Waste & $\begin{array}{l}\text { The stream contains the remaining from the } \\
\text { separation process }\end{array}$ \\
\hline Product & Stream containing the pure molybdenum disulfide \\
\hline
\end{tabular}

The simultaneous variation of both precursors follows a linear profile allowing the monitoring of the impact of each precursor on the final product amount as shown in Figure 5. The variation of the sulfur amount and the production of molybdenum disulfide follow a general linear trend, while the variation of the ammonium heptamolybdate amount increases quickly to reach the maximum stable point. The production of increased molybdenum disulfide nanoparticles is related obviously to the increased amounts of the used starting precursors. Figure 5 indicates the presence of an optimal point corresponding to the maximal production of molybdenum disulfide of $0.25 \mathrm{~g}$ for $0.10 \mathrm{~g}$ of sulfur and $0.38 \mathrm{~g}$ of ammonium heptamolybdate. The obtained results revealed that both sulfur and ammonium heptamolybdate 
Table 5: Results of the simulation with Aspen Plus.

\begin{tabular}{|c|c|c|c|c|c|c|}
\hline Involved weight (g) & Input & R-output & CF-waste & C-output & Waste & Product \\
\hline Sulfur & 0.05 & 0.01 & 0.01 & $1.89 \times 10^{-4}$ & 0 & 0 \\
\hline Molybdenum disulfide & 0 & 0.10 & $1.60 \times 10^{-3}$ & 0.10 & 0 & 0.10 \\
\hline Hydrazine & 6.19 & 6.16 & 6.06 & 0.10 & 0.10 & 0 \\
\hline Nitrogen & 0 & 0.03 & 0.03 & $4.41 \times 10^{-4}$ & $4.41 \times 10^{-4}$ & 0 \\
\hline Ammonia & 0 & 0.01 & 0.01 & $8.97 \times 10^{-3}$ & $1.53 \times 10^{-4}$ & 0 \\
\hline Ammonium heptamolybdate & 0.14 & 0.03 & $4.98 \times 10^{-4}$ & 0.03 & 0.03 & 0 \\
\hline Ethylenediamine & 80.91 & 80.91 & 79.55 & 1.36 & 1.36 & 0 \\
\hline Water & 0 & 0.05 & 0.04 & $7.56 \times 10^{-4}$ & $7.56 \times 10^{-4}$ & 0 \\
\hline Ammonium carbonate & 0.14 & 0.14 & 0.14 & $2.35 \times 10^{-3}$ & $2.35 \times 10^{-3}$ & 0 \\
\hline
\end{tabular}

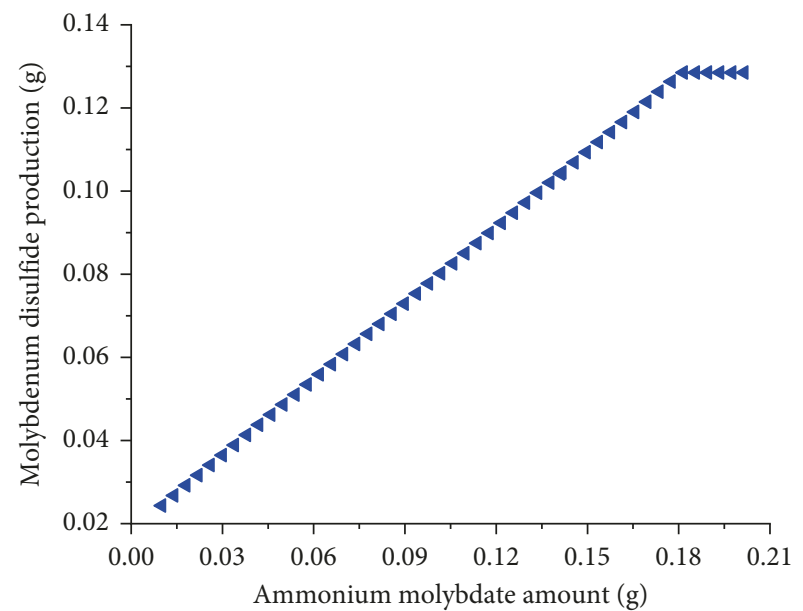

(a)

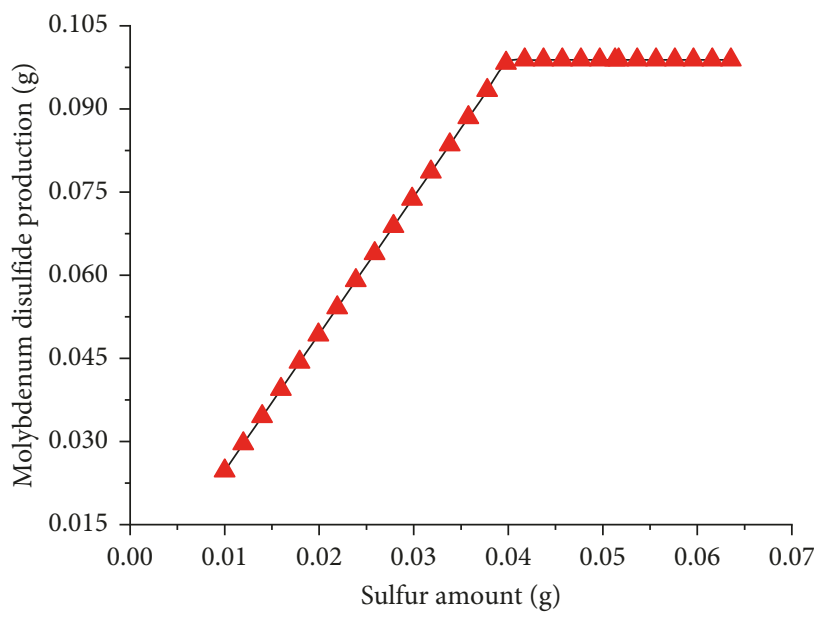

(b)

FIGURE 3: Effect of the precursor amount variation on the obtained molybdenum disulfide.

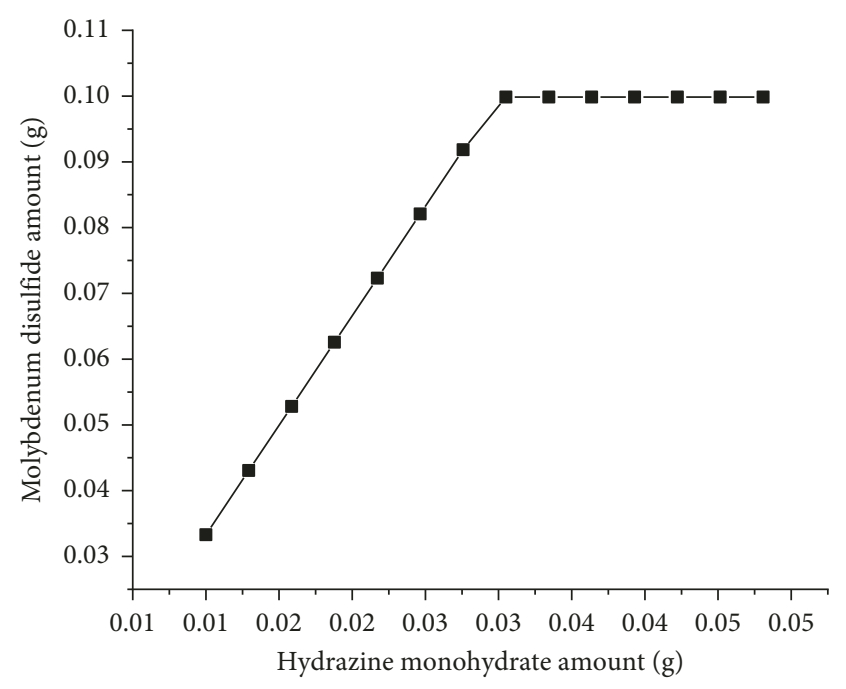

FIGURE 4: Effect of the hydrazine amount variation on the obtained molybdenum disulfide.

have similar influence on the production of molybdenum disulfide.

Figures 6 and 7 show that, as compared with the precursors, the hydrazine does not have an important effect on the amount of produced molybdenum disulfide, due to its

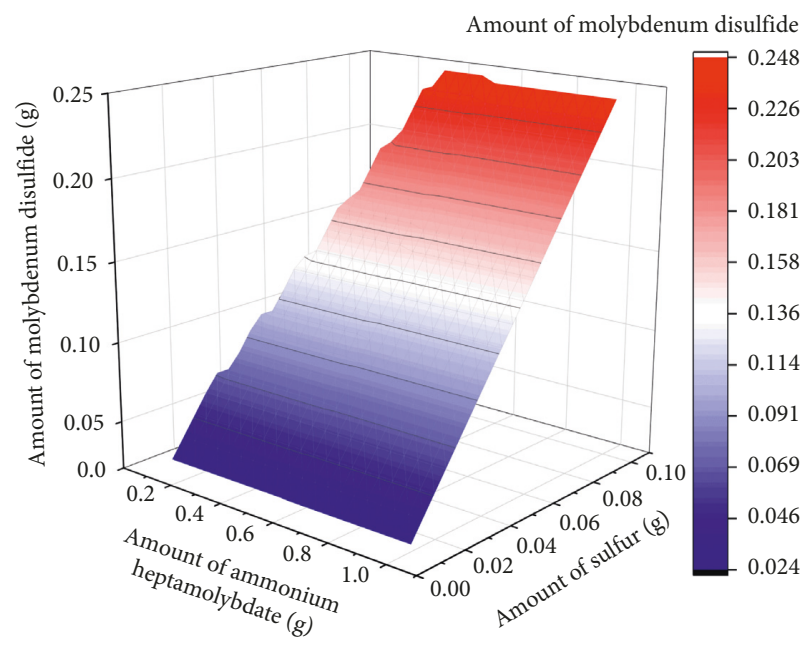

FIGURE 5: Effect of the simultaneous variation of the precursor amount on the obtained amount of molybdenum disulfide nanoparticles.

low reactivity in agreement with its role as a reducing agent. Indeed, the obtained result revealed that molybdenum disulfide production is proportional to the amount of ammonium heptamolybdate and sulfur, as shown in Figures 6 and 7 , respectively. This finding proves the stability of the 


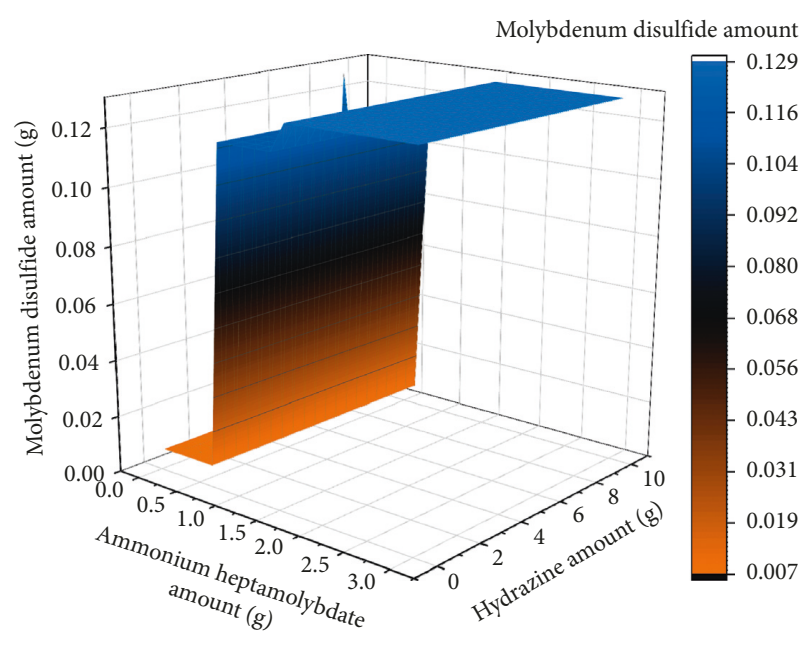

FIGURE 6: Effect of the simultaneous variation of the hydrazine and ammonium heptamolybdate amount on the obtained amount of molybdenum disulfide.

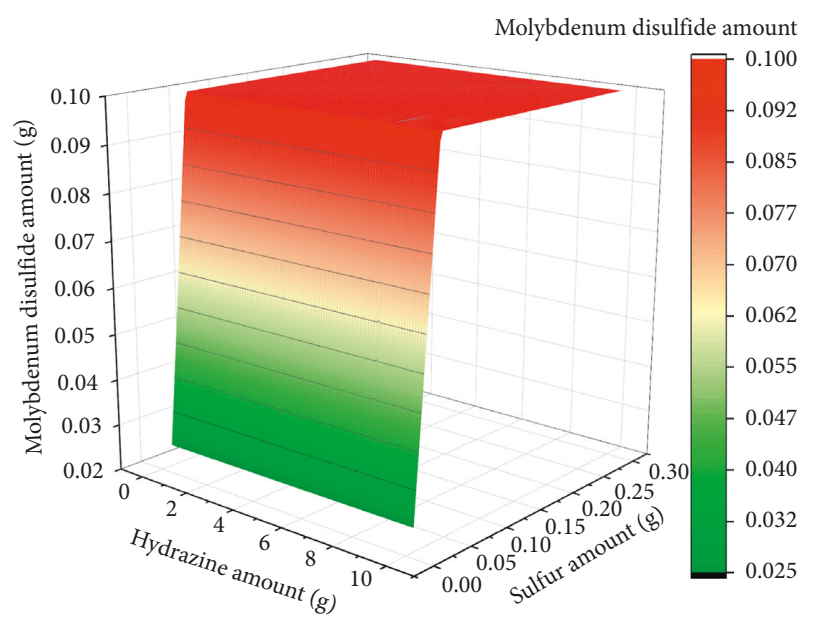

FIGURE 7: Effect of the simultaneous variation of the hydrazine and sulfur amount on the obtained amount of molybdenum disulfide.

adopted approach that might be useful for simulation of the product yield by the process at a larger scale.

\section{Conclusions}

In this paper, Aspen Plus software was used for simulation and sensitivity analysis of molybdenum disulfide synthesis process. The simulation was based on the result obtained by designed experiments carried at our laboratory, alongside the software's database and property methods. Interestingly, simulated results were found to be in good agreement with our experimental finding. The sensitivity analysis revealed that sulfur, ammonium heptamolybdate, and hydrazine have comparable effects on molybdenum disulfide production, whereas the simultaneous variation of the involved components does not have similar impact. The adopted approach shows quite interesting modelling stability that might pave the way to the scaling-up investigation of molybdenum disulfide at larger scale, although more accurate simulation might require further thermodynamic and kinetic investigations.

\section{Data Availability}

The underlying data can be provided upon request by email to mhachhach@uae.ac.ma.

\section{Conflicts of Interest}

The authors declare that there are no conflicts of interest regarding the publication of this paper.

\section{Acknowledgments}

Mouad Hachhach would like to thank Juan Ramón Portela Miguélez from the University of Cadiz for his help and guidance during his stay at the Laboratory of Analysis and Design of Supercritical Fluid Processes. The technical support of the University of Cadiz is also recognized for allowing us to use Aspen Plus. The Institute IRESEN/Morocco funded this research under the Project Innowind 13 Nanolubricant.

\section{References}

[1] N. Ye, T. Yan, Z. Jiang, W. Wu, and T. Fang, "A review: conventional and supercritical hydro/solvothermal synthesis of ultrafine particles as cathode in lithium battery," Ceramics International, vol. 44, no. 5, pp. 4521-4537, 2018.

[2] K. Zhang, J. M. Suh, J.-W. Choi, H. W. Jang, M. Shokouhimehr, and R. S. Varma, "Recent advances in the nanocatalyst-assisted $\mathrm{NaBH}_{4}$ reduction of nitroaromatics in water," ACS Omega, vol. 4, no. 1, pp. 483-495, 2019.

[3] M. Shokouhimehr, M. S. Asl, and B. Mazinani, "Modulated large-pore mesoporous silica as an efficient base catalyst for the henry reaction," Research on Chemical Intermediates, vol. 44, no. 3, pp. 1617-1626, 2017.

[4] K. Zhang, J. M. Suh, T. H. Lee et al., "Copper oxide-graphene oxide nanocomposite: efficient catalyst for hydrogenation of nitroaromatics in water," Nano Convergence, vol. 6, no. 1, 2019.

[5] A. Ahadi, S. Rostamnia, P. Panahi et al., "Palladium comprising dicationic bipyridinium supported periodic mesoporous organosilica (PMO): Pd@Bipy-PMO as an efficient hybrid catalyst for Suzuki-Miyaura cross-coupling reaction in water," Catalysts, vol. 9, no. 2, p. 140, 2019.

[6] S. M. Rafiaei, A. Kim, and M. Shokouhimehr, "Gadolinium triflate immobilized on magnetic nanocomposites as recyclable lewis acid catalyst for acetylation of phenols," Nanoscience and Nanotechnology Letters, vol. 6, no. 4, pp. 309-313, 2014.

[7] M. Nasrollahzadeh, M. Sajjadi, M. R. Tahsili, M. Shokouhimehr, and R. S. Varma, "Synthesis of 1substituted 1H-1,2,3,4-Tetrazoles using biosynthesized Ag/ sodium borosilicate nanocomposite," ACS Omega, vol. 4, no. 5, pp. 8985-9000, 2019.

[8] E. G. Vaschetto, S. G. Casuscelli, and G. A. Eimer, "Acidity versus catalytic activity in bi-structured nanomaterials," Microporous and Mesoporous Materials, vol. 268, pp. 170-177, 2018. 
[9] A. Kim, S. M. Rafiaei, S. Abolhosseini, and M. Shokouhimehr, "Palladium nanocatalysts confined in mesoporous silica for heterogeneous reduction of nitroaromatics," Energy and Environment Focus, vol. 4, no. 1, pp. 18-23, 2015.

[10] K. Zhang, K. Hong, J. M. Suh et al., "Facile synthesis of monodispersed Pd nanocatalysts decorated on graphene oxide for reduction of nitroaromatics in aqueous solution," Research on Chemical Intermediates, vol. 45, no. 2, pp. 599$611,2018$.

[11] M. Shokouhimehr, "Magnetically separable and sustainable nanostructured catalysts for heterogeneous reduction of nitroaromatics," Catalysts, vol. 5, no. 2, pp. 534-560, 2015.

[12] Q.-V. Le, G. Yang, Y. Wu, H. W. Jang, M. Shokouhimehr, and Y.-K. Oh, "Nanomaterials for modulating innate immune cells in cancer immunotherapy," Asian Journal of Pharmceutical Sciences, vol. 14, no. 1, pp. 16-29, 2019.

[13] A. Alam, Y. Zhang, H.-C. Kuan, S.-H. Lee, and J. Ma, "Polymer composite hydrogels containing carbon nanomaterials-morphology and mechanical and functional performance," Progress in Polymer Science, vol. 77, pp. 1-18, 2018.

[14] J. Sun, X. Li, W. Guo et al., "Synthesis methods of two-dimensional $\mathrm{MoS}_{2}$ : a brief review," Crystals, vol. 7, no. 7, p. 198, 2017.

[15] H. Akram, C. Mateos-Pedrero, E. Gallegos-Suárez, A. Guerrero-Ruíz, T. Chafik, and I. Rodríguez-Ramos, "Effect of electrolytes nature and concentration on the morphology and structure of $\mathrm{MoS}_{2}$ nanomaterials prepared using one-pot solvothermal method," Applied Surface Science, vol. 307, pp. 319-326, 2014.

[16] H. Yang, J. Zhao, C. Wu, C. Ye, D. Zou, and S. Wang, "Facile synthesis of colloidal stable $\mathrm{MoS}_{2}$ nanoparticles for combined tumor therapy," Chemical Engineering Journal, vol. 351, pp. 548-558, 2018.

[17] H. Akram, C. Mateos-Pedrero, E. Gallegos-Suárez et al., "Low solvothermal synthesis and characterization of hollow nanospheres molybdenum sulfide," Journal of Nanoscience and Nanotechnology, vol. 12, no. 8, pp. 6679-6685, 2012.

[18] H. Akram, O. Achak, S. Haffane et al., "Comparison of synthesis routes of inorganic fullerene-like nano-additives for wind turbine lubrication: application of life cycle assessment approach," in Proceedings of the 2014 International Renewable and Sustainable Energy Conference (IRSEC), pp. 788-790, Ouarzazate, Morocco, October 2014.

[19] Z. He and W. Que, "Molybdenum disulfide nanomaterials: structures, properties, synthesis and recent progress on hydrogen evolution reaction," Applied Materials Today, vol. 3, pp. 23-56, 2016.

[20] H. Shi, X. Fu, X. Zhou, D. Wang, and Z. Hu, "A low-temperature extraction-solvothermal route to the fabrication of micro-sized $\mathrm{MoS}_{2}$ spheres modified by Cyanex 301," Journal of Solid State Chemistry, vol. 179, no. 6, pp. 1690-1697, 2006.

[21] G. Li, C. Li, H. Tang et al., "Synthesis and characterization of hollow $\mathrm{MoS}_{2}$ microspheres grown from $\mathrm{MoO}_{3}$ precursors," Journal of Alloys and Compounds, vol. 501, no. 2, pp. 275-281, 2010.

[22] J. Zhao, P. Xie, C. Ye et al., "Outside-in synthesis of mesoporous silica/molybdenum disulfide nanoparticles for antitumor application," Chemical Engineering Journal, vol. 351, pp. 157-168, 2018.

[23] M. Zare, K. Namratha, K. Byrappa, D. M. Surendra, S. Yallappa, and B. Hungund, "Surfactant assisted solvothermal synthesis of $\mathrm{ZnO}$ nanoparticles and study of their antimicrobial and antioxidant properties," Journal of Materials Science \& Technology, vol. 34, no. 6, pp. 1035-1043, 2018.
[24] H. Akram, C. Mateos-Pedrero, E. Gallegos-Suarez, T. Chafik, A. Guerrero-Ruiz, and I. Rodríguez-Ramos, "Effect of surfactant concentration on the morphology of $\mathrm{Mo}_{\mathrm{x}} \mathrm{S}_{\mathrm{y}}$ nanoparticles prepared by a solvothermal route," Green Processing and Synthesis, vol. 6, no. 2, pp. 161-171, 2016.

[25] M. Puig-Gamero, J. Argudo-Santamaria, J. L. Valverde, P. Sánchez, and L. Sanchez-Silva, "Three integrated process simulation using aspen plus $^{\circledR}$ : pine gasification, syngas cleaning and methanol synthesis," Energy Conversion and Management, vol. 177, pp. 416-427, 2018.

[26] Y. M. Camacho, S. Bensaid, S. Lorentzou et al., "Development of a robust and efficient biogas processor for hydrogen production. Part 1: modelling and simulation," International Journal of Hydrogen Energy, vol. 42, no. 36, pp. 22841-22855, 2017.

[27] C. Somers, A. Mortazavi, Y. Hwang, R. Radermacher, P. Rodgers, and S. Al-Hashimi, "Modeling water/lithium bromide absorption chillers in ASPEN Plus," Applied Energy, vol. 88, no. 11, pp. 4197-4205, 2011.

[28] H. Weifeng, S. Hongye, M. Shengjing, and C. Jian, "Multiobjective optimization of the industrial naphtha catalytic reforming process," Chinese Journal of Chemical Engineering, vol. 15, no. 1, pp. 75-80, 2007.

[29] I. Rossetti, N. Pernicone, F. Ferrero, and L. Forni, "Kinetic study of ammonia synthesis on a promoted $\mathrm{Ru} / \mathrm{C}$ catalyst," Industrial \& Engineering Chemistry Research, vol. 45, no. 12, pp. 4150-4155, 2006.

[30] L. Shen, Y. Gao, and J. Xiao, "Simulation of hydrogen production from biomass gasification in interconnected fluidized beds," Biomass and Bioenergy, vol. 32, no. 2, pp. 120-127, 2008.

[31] M. Garcia, H. K. Knuutila, and S. Gu, "ASPEN PLUS simulation model for $\mathrm{CO}_{2}$ removal with MEA: validation of desorption model with experimental data," Journal of Environmental Chemical Engineering, vol. 5, no. 5, pp. 4693-4701, 2017.

[32] F. Larbi, A. García, L. J. Del Valle et al., "Simulation basis for a techno-economic evaluation of chitin nanomaterials production process using Aspen Plus ${ }^{\circledR}$ software," Data in Brief, vol. 20, pp. 1556-1560, 2018.

[33] A. Al-Atta, T. Huddle, Y. G. Rodríguez et al., "A technoeconomic assessment of the potential for combining supercritical water oxidation with "in-situ" hydrothermal synthesis of nanocatalysts using a counter current mixing reactor," Chemical Engineering Journal, vol. 344, pp. 431-440, 2018.

[34] G. Demazeau, "Solvothermal reactions: an original route for the synthesis of novel materials," Journal of Materials Science, vol. 43, no. 7, pp. 2104-2114, 2008.

[35] L. F. Albright, Albrights Chemical Engineering Handbook, CRC Press, Boca Raton, FL, USA, 2009.

[36] C.-C. Chen, "Representation of solid-liquid equilibrium of aqueous electrolyte systems with the electrolyte NRTL model," Fluid Phase Equilibria, vol. 27, pp. 457-474, 1986.

[37] B. Mock, L. B. Evans, and C.-C. Chen, "Thermodynamic representation of phase equilibria of mixed-solvent electrolyte systems," AIChE Journal, vol. 32, no. 10, pp. 1655-1664, 1986.

[38] G. Brunner, "Calculation of phase equilibria and their relation to separation with supercritical fluids," The Journal of Supercritical Fluids, vol. 134, pp. 2-11, 2018.

[39] A. C. Dimian, C. S. Bildea, and A. A. Kiss, Integrated Design and Simulation of Chemical Processes, Elsevier, Amsterdam, Netherlands, 2014

[40] R. Schefflan, Teach Yourself the Basics of Aspen Plus ${ }^{\mathrm{TM}}$, WileyBlackwell, Hoboken, NJ, USA, 2011. 
[41] H. S. Fogler and N. M. Gurmen, Aspen Plus ${ }^{\mathrm{TM}}$ Workshop for Reaction Engineering and Design, The University of Michigan, Ann Arbor, MI, USA, 2002.

[42] J. P. Chen and L. L. Lim, "Key factors in chemical reduction by hydrazine for recovery of precious metals," Chemosphere, vol. 49, no. 4, pp. 363-370, 2002.

[43] A. Hemmati and H. Rashidi, "Mass transfer investigation and operational sensitivity analysis of amine-based industrial $\mathrm{CO}_{2}$ capture plant," Chinese Journal of Chemical Engineering, vol. 27, no. 3, pp. 534-543, 2018. 


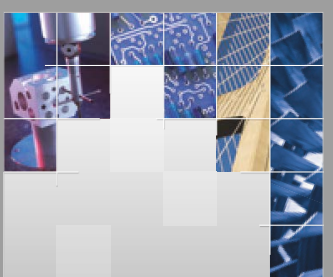

\section{Enfincering}
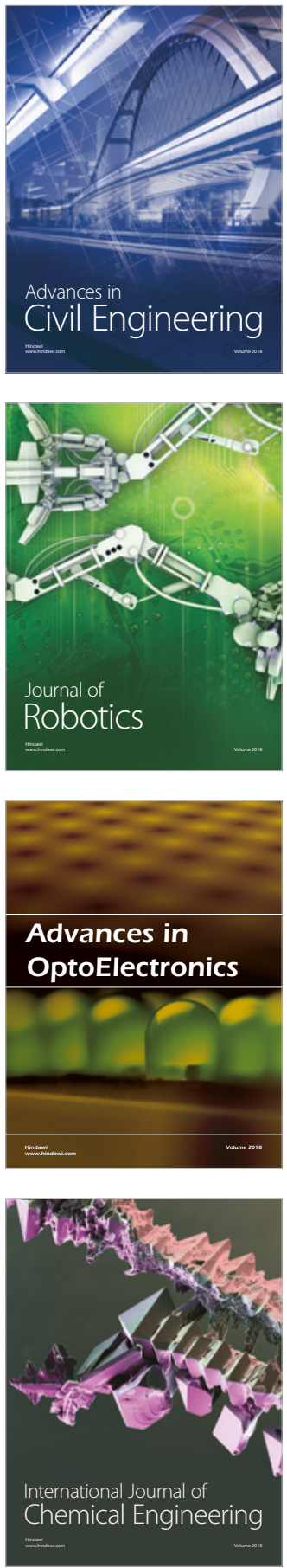

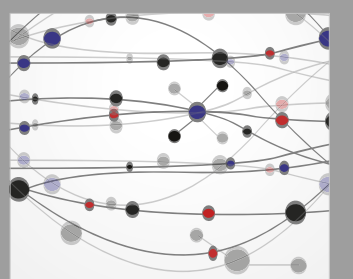

\section{Rotating \\ Machinery}

The Scientific World Journal

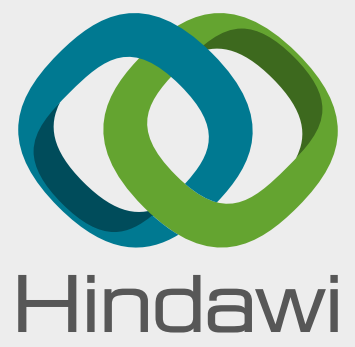

Submit your manuscripts at

www.hindawi.com
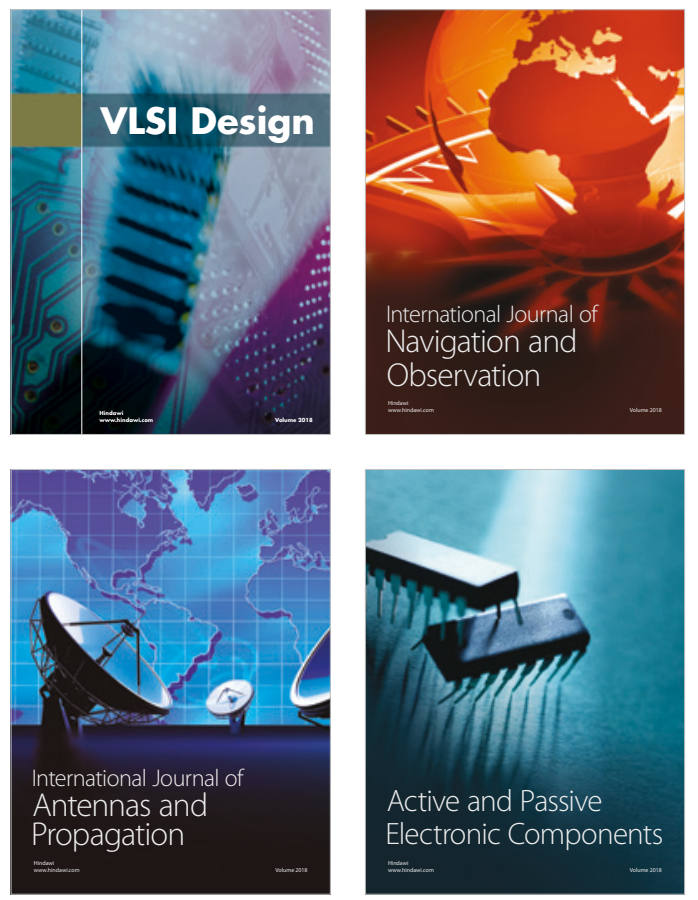
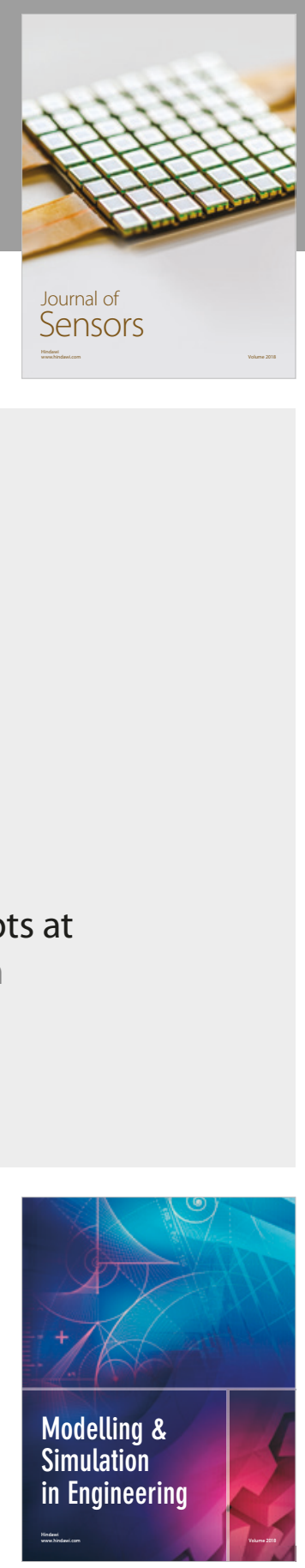

\section{Advances \\ Multimedia}
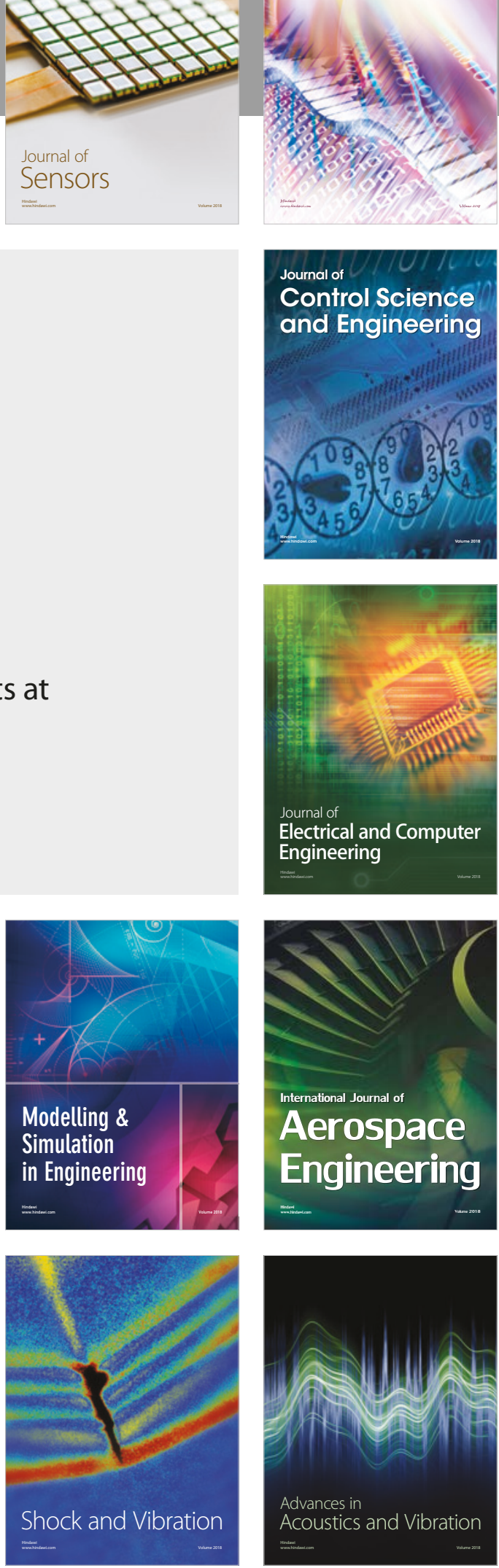